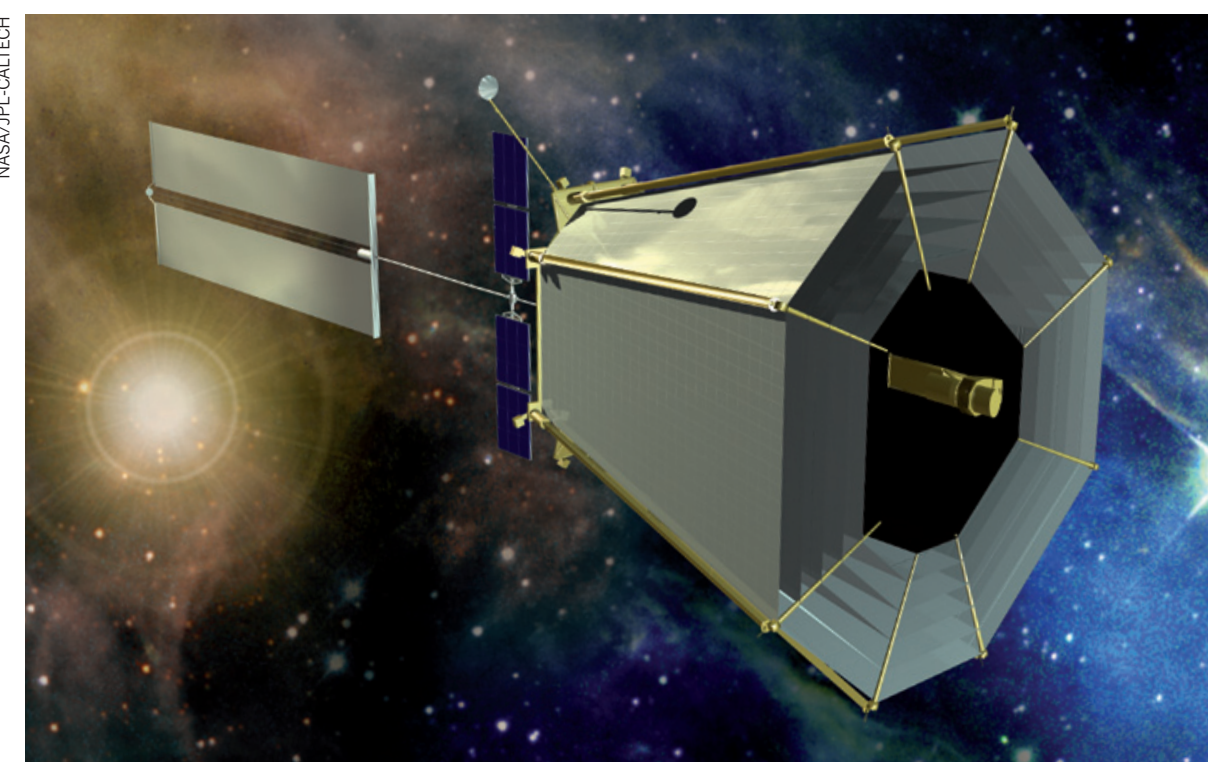

Exoplanet hunters want something to replace the postponed Terrestrial Planet Finder.

SPACE SCIENCE

\title{
Astronomers mull merger of missions
}

\section{Cosmic-origins scientists convene with exoplanet hunters.}

\section{BY EUGENIE SAMUEL REICH}

$\mathrm{N}$ ASA's constrained budget is encouraging some creative pairings. This week, scientists eager to find other habitable worlds explored the possibility that a future space telescope for probing the origins of stars and galaxies could serve their needs as well.

For months, NASA's Cosmic Origins Program Analysis Group (COPAG) has been discussing the prospects for a large ultraviolet-visiblewavelength telescope that would fly sometime after 2020 and explore the architecture of galaxies and the details of their formation. Then the Exoplanet Exploration Program Analysis Group (ExoPAG) got wind of the plan. Astronomer Kenneth Sembach of the Space Telescope Science Institute in Baltimore,
Maryland, says that he was originally expecting a small group at a 26 April joint COPAG and ExoPAG meeting he is co-hosting. In the end, 49 astronomers attended in person, online or by dial-in. "It's taken on a life of its own," he says.

Exoplanet hunters are keen for a new instrument. Results are pouring in from Kepler, NASA's first orbiting telescope dedicated to finding other Earths. Although Kepler can detect planets between about 184 and 920 parsecs away that are transiting in front of their host stars, it is generally unable to determine their masses or compositions. Researchers hope for a follow-up mission that would image Earth-like planets around the nearest 100 Sun-like stars at sufficient resolution to detect traces of oxygen or water in their atmospheres.

The Terrestrial Planet Finder - a NASA project along these lines - was indefinitely postponed in 2007, but the agency has continued to put US\$6 million a year into developing technology for exoplanet searches. Now planet hunters think that joining forces with COPAG will be the winning strategy. Jim Kasting, a planetary scientist at Pennsylvania State University in University Park who is chair of the exoplanet group, says the two communities would both like to see a 4-8-metre telescope in space that would cost in excess of $\$ 5$ billion. "Our interests are basically aligned," he says.

Such a mission would compete for top billing in the next decadal survey of astronomy by the US National Academy of Sciences, due in 2020. The big question, which follow-up meetings will consider, is whether the same technology can do both kinds of science.

A cosmic-origins mission would need to collect as much ultraviolet and visible light as possible to image intergalactic gas, star formation and Sun-like stars in nearby galaxies. A planet-hunting probe would need a coronagraph to block direct light from host stars, and would have to be sensitive to the visible and near-infrared wavelengths that Earth-like planets primarily emit. The infrared 6.5-metre James Webb Space Telescope (JWST), scheduled for launch in 2014, will be able to see larger planets but will not be sensitive to Earth-sized ones. The key to making the joint concept work will be developing a reflective coating for the telescope's mirror that works from the ultraviolet to the infrared ranges and does not distort the incoming light waves in a way that would make it difficult to tease out the image of an exo-Earth.

Sara Seager, a planet hunter at the Massachusetts Institute of Technology in Cambridge, is not convinced by the strategy. "It sounds greedy, but you're cheating yourself if you make a mission everyone has to share," she says. Although smaller-scale planet-hunting missions set to launch in the next decade will not be sensitive enough to find other Earths, she adds, they may help increase momentum to build a dedicated space telescope that can.

But Natalie Batalha of San José State University in California, deputy team leader for Kepler, says a joint mission may be the logical response to NASA's money troubles, which cost overruns of the JWST have exacerbated. "These projects are very expensive and ambitious and we have to find ways to leverage off other programmes," she says.

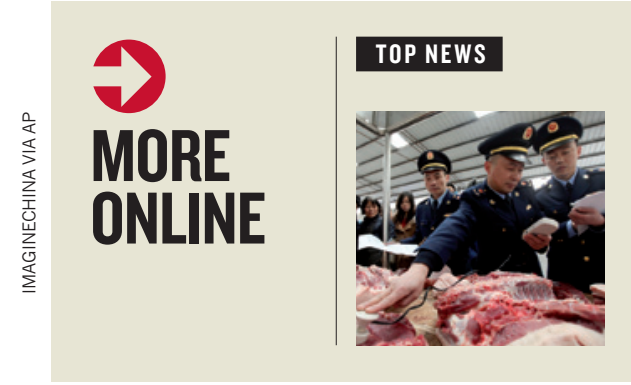

China hopes research centre can quell foodsafety fears go.nature.com/ kejnxy

\section{MORE NEWS}

- Fire ants form water-repellent rafts to escape floods go.nature.com/cmb7im - Effects of quantum entanglement seen with human eyes go.nature.com/ q7mo98

- New drug targets raise hopes for hepatitis $C$ cure go.nature.com/scwtof

\section{VIDEO}

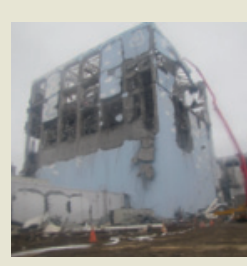

The Fukushima nuclear crisis explained in less than 5 minutes go.nature.com/ ulbodm 